\title{
Vacancy engineered ceria nanostructures for protection from radiation-induced cellular damage
}

Roy W. Tarnuzzer, Jimmie Colon, Swanand Patil, and Sudipta Seal

\section{Supporting Information}

\author{
Materials and Methods.
}

Cell Lines and Reagents. CRL-8798, an immortalized normal breast epithelial cell line, and MCF-7, breast carcinoma cell line, were obtained from American Type Culture Collection (Manassas, VA). Cell lines were maintained in a growth medium comprised of the following constituents: minimum essential medium (Eagle) with 2mM l-glutamine and Earle's BSS adjusted to contain $1.5 \mathrm{~g} / \mathrm{L}$ sodium bicarbonate, $0.1 \mathrm{mM}$ non-essential amino acids, and $1.0 \mathrm{mM}$ sodium pyruvate, $90 \%$; fetal bovine serum, $10 \%$ from Invitrogen Corporation (Carlsbad, CA). The cells were incubated at $37^{\circ} \mathrm{C}$, and $5 \% \mathrm{CO}_{2}$.

Cerium Oxide Nanoparticle Synthesis and Characterization. Cerium oxide nanoparticles were prepared by the microemulsion method. The nano-sized micelles in the microemulsion system act as nano-reactors for the nanoparticle formation. The microemulsion system consisted of the surfactant, sodium bis(2-ethylhexyl) sulphosuccinate (AOT), toluene and water. All the chemicals were purchased from Aldrich Chemicals Company, Inc. Details of the synthesis are published elsewhere ${ }^{1,2}$. The particles obtained in toluene were precipitated and washed with acetone and water for several times to remove the surfactant completely from the particles. The particles thus obtained were then re-dispersed in water. The solution was sterile filtered through a $0.2 \mu \mathrm{m}$ syringe filter for use in radioprotection experiments.

The particle morphology was studied using high resolution transmission electron microscopy (HRTEM), x-ray diffraction (XRD) was used for crystal structure studies and the surface chemistry of 
the ceria nanoparticles was studied using x-ray photoelectron spectroscopy (XPS). The Ceria nanoparticles were deposited on the carbon coated copper grid for HRTEM analysis by the dip coating method. The HRTEM images of the particles were obtained with a Philips (Tecnai Series) transmission electron microscope operating at $300 \mathrm{keV}$. The crystal structure information of the synthesized nanoceria was taken using XRD (Rigaku model) with $\mathrm{Cu} \mathrm{K}_{\alpha 1}$ radiation. The data was recorded at scan rate of 0.25 degree/min. The XPS data was obtained using a 5400 PHI ESCA (XPS) spectrometer. The base pressure during XPS analysis was $10^{-9}$ Torr and $\mathrm{Mg}-\mathrm{K}_{\alpha}$ X-ray radiation $(1253.6 \mathrm{eV})$ at a power of 200 watts was used. The binding energy of the $\mathrm{Au}\left(4 \mathrm{f}_{7 / 2}\right)$ at $84.0 \pm 0.1 \mathrm{eV}$ was used to calibrate the binding energy scale of the spectrometer. Any charging shift produced in the spectrum by the sample was carefully removed by taking $\mathrm{C}(1 \mathrm{~s})$ position $(284.6 \mathrm{eV})$ as a reference line as shown by Barr and Seal $^{3}$. XPS spectra smoothening and baseline subtraction was carried out using PeakFit (Version 4) software.

Radiation Treatment. Cell lines were plated at 5,000 to $1.3 \times 10^{6}$ cells per well in 96 or 6 well plates. Sterile filtered Cerium Oxide nanoparticles were added to each well at 10 or $100 \mathrm{nM}, 24$ hours prior to irradiation. Plates were irradiated at the dose of 0 to 10 Gy with a Varian 600C Single Beam Linear Accelerator (Palo Alto, CA). Plates were returned to an incubator and assayed for cell survival at 24 and 48 hours after irradiation.

MTT Assay. Cell viability was assayed by MTT assay as described in the manufacturer's protocol. Briefly, a $20 \mu \mathrm{l}$ of a $5 \mathrm{mg} / \mathrm{ml}$ solution of MTT (Promega Corporation, Madison, WI) was added to each well of a 96-well plate, 2 hours before the end of the incubation. The plates were incubated in a $\mathrm{CO}_{2}$ incubator at $37^{\circ} \mathrm{C}$ for 2 hours. The supernatant was aspirated and the cells dissolved in $200 \mu$ of DMSO by pipetting up and down. Plates were further incubated at room temperature for 2 hours. The plates were transferred to a plate reader and absorbance measured at $550 \mathrm{~nm}$.

TUNEL Assay. Cells were grown on chamber slides (Fisher Scientific, Hampton, NH) and pretreated with $10 \mathrm{nM}$ nanoceria for 24 hours. Slides were irradiated with $10 \mathrm{~Gy}$ and incubated at $37^{\circ} \mathrm{C}$ for 24 or 
48 hours. TUNEL staining was carried out as per the manufacturer's protocol (Chemicon International, Temacula, CA). Briefly, both slide adherent and suspended cells were collected and assayed for TUNEL staining by first fixing in 1\% paraformaldehyde. Endogenous peroxidase was quenched with $3 \% \mathrm{H} 2 \mathrm{O} 2$ for 5 minutes followed by washing in $\mathrm{dH} 2 \mathrm{O}$ for 5 minutes. Slides were incubated with terminal deoxynuclotidyl transferase with digoxigenin-linked dUTP for 1 hour at $37^{\circ} \mathrm{C}$. The reaction is stopped, slides washed and then incubated with anti-digoxigenin antibody conjugated with horseradish peroxidase for 30 minutes at room temperature in a humidified chamber. Slides were washed, incubated with the peroxidase substrate for 3-6 minutes, washed, coverslips mounted and viewed under a light microscope.

Statistical Analysis. All experiments were completed in triplicate and results expressed as Mean +/SD. Differences in radiosensitivity of cell lines and radioprotection of compounds was determined by a paired Student's T-test and a p-value equal to or less than 0.05 was considered statistically significant.

1. Patil, S.; Kuiry, S. C.; Seal, S.; Vanfleet, R. Journal of Nanoparticle Research. 2002. 4, 433.

2. Bailey, D.; Patil, S.; Seal, S.; Rzigalinski, B. Patent Pending, UCF 60/408,275 2003.

3. Barr, T. L.; Seal, S. Journal of Vacuum Science and Technology. 1995, 13A, 1239. 